\title{
TSD Study of Pure and Chloranil Doped Polystyrene Films Grown from Solutions
}

\author{
Y. K. KulShrestha* and A. P. SRivastV́a \\ Department of Physics, University of Saugar, (M.P.), India.
}

(Received October 12, 1978)

\begin{abstract}
Three peaks, namely, $\beta, \alpha$, and $\rho$ peaks, have been observed in the TSD spectra of commercial polystyrene (PS) at about 338,368 , and $418 \mathrm{~K}$ respectively. Their origin is due respectively to the trapping of charge carriers by the particular atoms or molecules pressent in the amorphous regions of polystyrene, to the segmental motion of main chains, and to the accumulation of space charges at the crystalline and amorphous interfaces. Doping of polystyrene with chloranil [electron affinity $1.4 \mathrm{eV}\left(2.24 \times 10^{-19} \mathrm{~J}\right)$ ] has been found to form charge-transfer complexes. This probably involves three mechanisms: increase in the mobility of charge carriers, reduction in the trapping sites for charges, and provision of conductive path ways for them through the crystallineamorphous interfaces.
\end{abstract}

KEY WORDS TSD / Electret / Activation Energy / Relaxation / Charge-

Transfer Complex / Space Charge / Polarisation / Depolarisation /

The thermally stimulated discharge (TSD) current technique has been widely applied to a number of inorganic and organic compounds, but its application to polymers has been rather limited. Since TSD is governed by relaxation phenomena specific to the polymers, it can be used to study molecular motions in them at low frequency, a study which is of paramount interest for several applications. This paper reports a systematic study of TSD of pure as well as of chloranil-doped films (about $25 \mu$ m thick) of polystyrene grown from solutions. The method is based on the measurement and interpretation of the discharge current which is generated on heating a polarised specimen (electret).

\section{EXPERIMENTAL}

Commercial polystyrene (PS) supplied by $\mathrm{M} / \mathrm{S}$. Impex Corp., Bombay was used for this investigation. Films of about $25 \mu \mathrm{m}$ in thickness were grown on the surface of cleaned microscopic glass slides by slowly pulling them out vertically from a solution of PS in cyclohexanone. The films were allowed to dry in air for about a week for complete vaporization of the solvent. Incompletely dried films

\footnotetext{
* Permanent address: Department of Physics,
} Dayanand Vedic College, ORAI (U.P.), India. were seen to give erroneous and nonreproducible results. Doping was done with chloranil by dissolving PS and chloranil together in cyclohexanone at room temperature over a period of days. It was found that doping takes about a week to complete. Three different concentrations of dopant, viz., 0.25 , 1 , and $4 \%$ by weight, were used. Films of doped PS were also prepared in the same manner as those of pure PS.

Vacuum deposited silver electrodes were used for the measurement in sandwich configuration. While the lower electrode was deposited on the glass slide itself before dipping it in the solution, the upper electrode was deposited over the film when it was completely dried. Figure 1 gives the geometry of the sandwich configuration of film and electrodes together. A piece of teflon was used to insulate the upper electrode from the glass slide so as to eliminate any possible leakage of current through the slide. Contacts were made by gently pressing two copper discs, as shown in Figure 1. In this way a pressureless contact was achieved. Current was measured with an electrometer amplifier supplied by $\mathrm{M} / \mathrm{S}$. Electronic Corporation of India Ltd.; this was carefully shielded and grounded. Before measurement, the films were kept short circuited in a desiccator for about $24 \mathrm{~h}$. 


\section{Y. K. Kulshrestha and A. P. Srivastv́a}

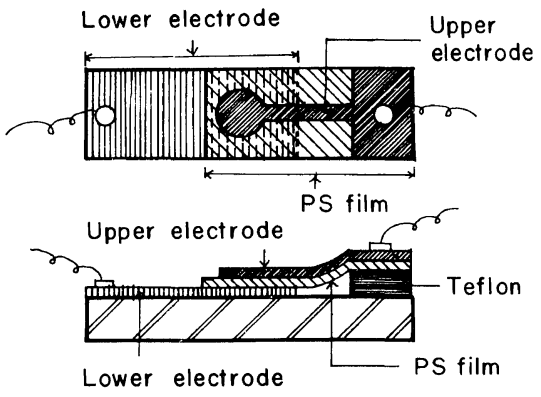

Figure 1. Geometry of sandwich configuration of PS film and electrodes.

The TSD cycle was obtained as follows. The sample was placed inside an oven whose temperature could be raised linearly by feeding a controlled voltage with the help of an autotransformer. First the oven and with it the sample was allowed to achieve an equilibrium temperature equal to the polarising temperature $\left(T_{\mathrm{p}}\right)$, which was kept at $403 \mathrm{~K}$ each time. This temperature was chosen because it has been reported ${ }^{1}$ that a space-charge peak can be obtained only when the polarising temperature is well above the glass-transition temperature of the dielectric, which has been reported ${ }^{2}$ to be $373 \mathrm{~K}$ for PS. Ad.c. voltage $\left(V_{\mathrm{p}}\right)$ was applied for $45 \mathrm{~min}$ at the temperature $T_{\mathrm{p}}$ using batteries. The sample was then cooled to room temperature, with the field still applied, within $15 \mathrm{~min}$. Then the field was switched off and the sample was short circuited for an arbitrary time of $5 \mathrm{~min}$, so as to remove frictional and stray surface charges, if any. ${ }^{3}$ Thereafter the sample was heated at a uniform rate of $276 \mathrm{~K} / \mathrm{min}$ from room temperature to about $453 \mathrm{~K}$ and the current was recorded. The temperature of the sample was measured with a precalibrated precision copperconstantin thermocouple attached close to it.

\section{THEORY}

According to Perlmann and Creswell, ${ }^{4}$ the discharge current at any time $t$, due to the release of trapped charges on heating the polarised specimen linearly, is given by

$$
j(t)=A \exp \left[-W / k T-B \int_{T_{0}}^{T} \exp (-W / k T) \mathrm{d} T\right]
$$

where $A$ and $B$ are constants, $W$ is the activation energy, $k$ is Boltzmann's constant, $T_{0}$ is room temperature, and $T$ is the temperature attained by the specimen in time $t$ on heating it at a constant uniform rate $\beta$.

The total charge released is given by

$$
Q=\int_{0}^{\infty} j(t) \mathrm{d} t
$$

and can be calculated by the area under the TSD curve. The temperature at which the maximum current occurs can be obtained from the following equation.

$$
\tau_{0}=\frac{k T_{\mathrm{m}}{ }^{2}}{\beta W \exp \left(W / k T_{\mathrm{m}}\right)}
$$

The above expression has been obtained by differentiating eq 1 and equating $\mathrm{d} j / \mathrm{d} T$ to zero after substituting the values of contants $A$ and $B$. It is evident from eq 3 that $T_{\mathrm{m}}$, i.e., the peak temperature, is independent of both the polarising field $E_{\mathrm{p}}$ and the polarising temperature $T_{\mathrm{p}}$, but depends upon the rate of heating.

The activation energy $W$ can be determined from the low-temperature tail of eq 1 using

$$
\ln j=\ln A-W / k T
$$

Thus a plot of $\ln j v s .1 / T$ gives a straight line, from the slope of which the value of $W$ can be calculated. This is called the initial rise method of Garlick and Gibson. ${ }^{5}$

\section{RESULTS}

TSD spectra of pure commercial PS at different forming voltages are shown in Figure 2. It can be seen that three peaks appear: the first at about $338 \mathrm{~K}$, the second at about $368 \mathrm{~K}$, and the third at about $418 \mathrm{~K}$. The positions of these peaks are found to be almost independent of the forming field. Slight variations in peak positions might be due to slight variations in heating rate. ${ }^{6}$ It can be further seen that these peaks are of increasing magnitude with the increasing polarising field. We call the first peak $\beta$, the second $\alpha$, and the third $\rho . \alpha$ and $\rho$ peaks are well defined, while $\beta$ peak is resolved only at 72 volts. Activation energies for the three peaks were calculated by the initial rise method from the plots of $\log j$ vs. $10^{3} / T$ (Figures 3, 4, and 5). Relaxation times $\tau_{0}$, $\tau_{300}$, and $\tau_{T_{\mathrm{m}}}$ at infinite temperature, room tempera- 


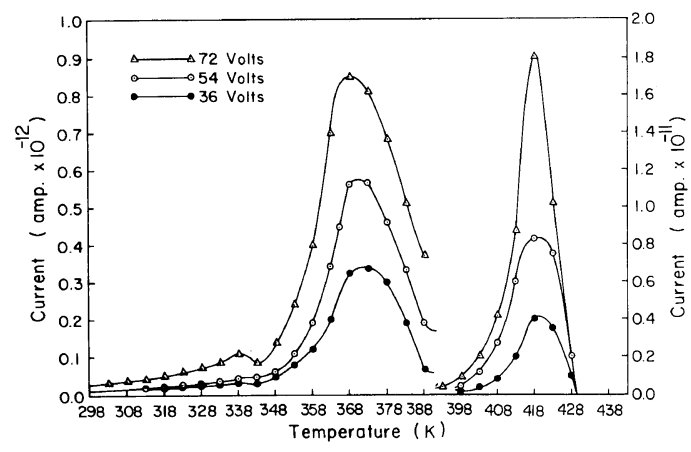

Figure 2. TSD current $v s$. temperature for undoped PS at three different polarising fields. Vertical scale for $\beta$ and $\alpha$ peaks should be read on left handed side and for $\rho$ peak on right handed side.

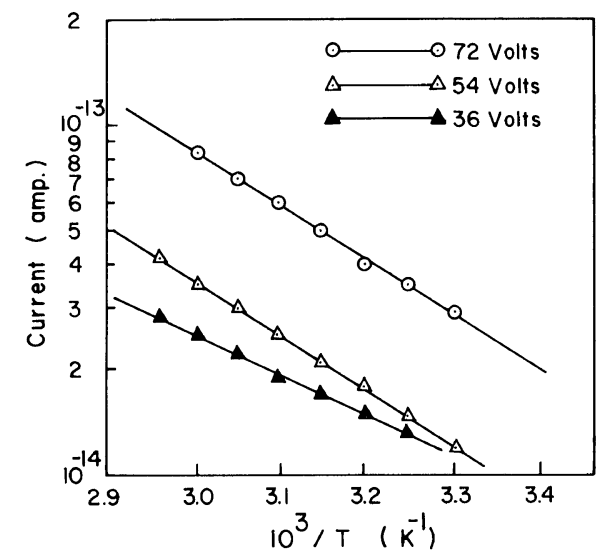

Figure 3. TSD current vs. $10^{3} / T$ plots for $\beta$ peaks.

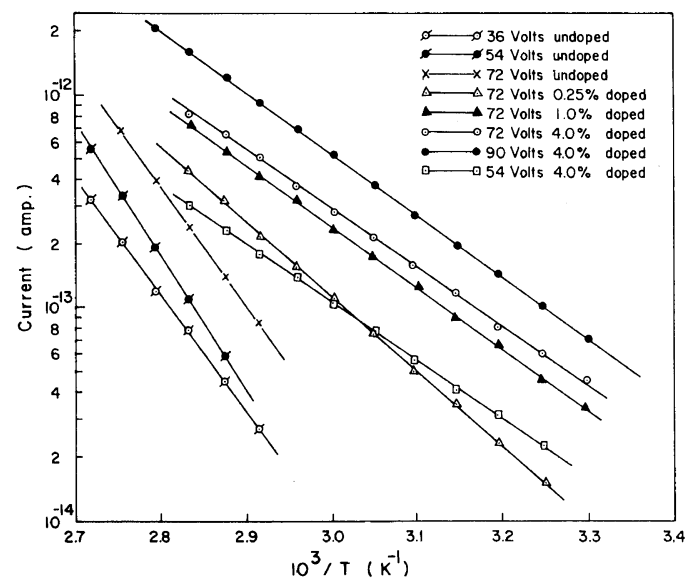

Figure 4. TSD current $v s .10^{3} / T$ for plots for $\alpha$ peaks.

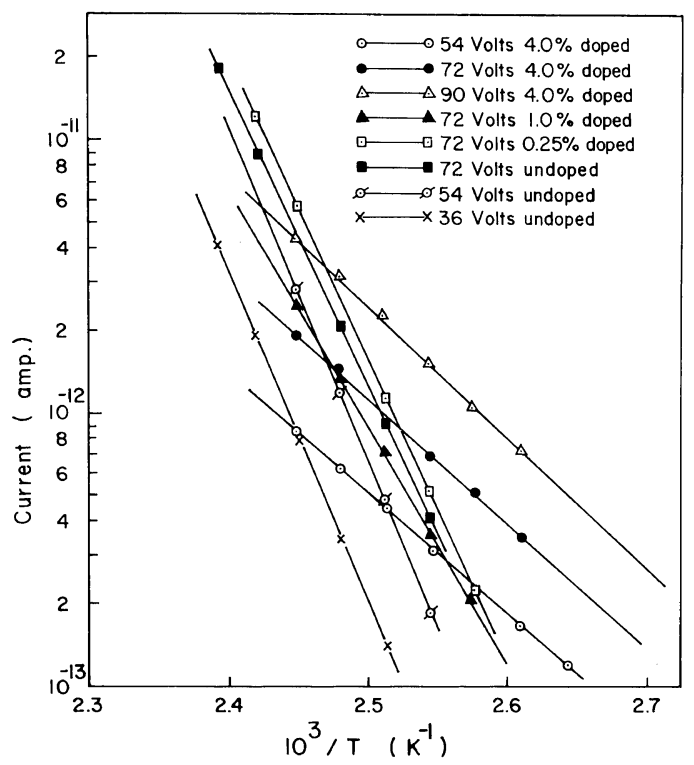

Figure 5. TSD current $v s$. $10^{3} / T$ plots for $\rho$ peaks.

ture $(300 \mathrm{~K})$ and peak temperature $\left(T_{\mathrm{m}}\right)$ respectively were also calculated. ${ }^{4}$ Their average values have been summarised in Table I.

Figure 6 represents the TSD curves for chloranildoped PS for different dopant concentrations along with that for pure PS under identical conditions. It can be seen that in the thermograms of doped PS, only the two peaks $\alpha$ and $\rho$ are obtained, the $\beta$ peak being completely absent. It can be further seen that there are no significant variations in the positions or in the magnitudes of $\alpha$ and $\rho$ peaks in the case of $0.25 \%$-doped PS from that in pure PS. However, for higher concentrations of dopants (i.e., 1 and $4 \%$ ) the positions of both $\alpha$ and $\rho$ peaks suffer a shift of about $10 \mathrm{~K}$ towards the lower temperature side. At the same time, the magnitudes of $\alpha$ peaks are more or less the same in all the cases, but the magnitudes of $\rho$ peaks decrease with increasing dopant concentration. Activation energies calculated from the plots of $\log j v s .10^{3} / T$ (Figures 4 and 5) show a decrease with increasing dopant concentration for both $\alpha$ and $\rho$ peaks. All these quantities, including relaxation times, have been included in Table I.

Figure 7 represents the TSD curves for $4 \%$ doped PS at different polarising voltages. It is seen that the position of $\alpha$ and $\rho$ peaks in this case too remain unchanged, but the magnitudes go on increasing with increasing polarising voltages. 
Y. K. Kulshrestha and A. P. SRivastv́a

Table I. Relaxation times for PS samples at infinite temperature, room temperature, and peak temperature

\begin{tabular}{|c|c|c|c|c|c|}
\hline \multirow[t]{2}{*}{ Sample } & $\begin{array}{l}\text { Activation } \\
\text { energy }\end{array}$ & $T_{\mathrm{m}}$ & $\tau_{0}$ & $\tau_{300}$ & $\tau_{\tau_{\mathrm{m}}}$ \\
\hline & $\mathrm{eV}^{\mathrm{a}}$ & K & s & s & s \\
\hline \multicolumn{6}{|c|}{$\beta$ PEAK } \\
\hline Undoped & $0.23 \pm 0.02$ & $338 \pm 2$ & $(3.0 \pm 0.5) \times 10^{-1}$ & $(2.2 \pm 0.3) \times 10^{3}$ & $(8.1 \pm 0.5) \times 10^{2}$ \\
\hline \multicolumn{6}{|c|}{$\alpha$ PEAK } \\
\hline Undoped & $1.13 \pm 0.02$ & $368 \pm 2$ & $(5.0 \pm 0.2) \times 10^{-13}$ & $(9.0 \pm 0.5) \times 10^{5}$ & $(2.8 \pm 0.2) \times 10^{2}$ \\
\hline $25 \%$ doped & $0.72 \pm 0.02$ & $368 \pm 2$ & $(2.3 \pm 0.2) \times 10^{-8}$ & $(1.7 \pm 0.3) \times 10^{4}$ & $(1.1 \pm 0.3) \times 10^{2}$ \\
\hline $1 \%$ doped & $0.58 \pm 0.01$ & $358 \pm 2$ & $(2.6 \pm 0.2) \times 10^{-6}$ & $(1.6 \pm 0.2) \times 10^{4}$ & $(4.2 \pm 0.2) \times 10^{2}$ \\
\hline $4 \%$ doped & $0.57 \pm 0.01$ & $358 \pm 2$ & $(5.2 \pm 0.2) \times 10^{-6}$ & $(1.5 \pm 0.2) \times 10^{4}$ & $(4.3 \pm 0.1) \times 10^{2}$ \\
\hline \multicolumn{6}{|c|}{$\rho$ PEAK } \\
\hline Undoped & $2.3 \pm 0.05$ & $418 \pm 2$ & $(3.8 \pm 0.1) \times 10^{-26}$ & $(3.2 \pm 0.2) \times 10^{13}$ & $(1.5 \pm 0.1) \times 10^{2}$ \\
\hline $25 \%$ doped & $2.2 \pm 0.07$ & $418 \pm 2$ & $(4.5 \pm 0.2) \times 10^{-25}$ & $(3.5 \pm 0.1) \times 10^{12}$ & $(1.3 \pm 0.1) \times 10^{2}$ \\
\hline $1 \%$ doped & $1.67 \pm 0.03$ & $408 \pm 2$ & $(4.4 \pm 0.1) \times 10^{-19}$ & $(4.5 \pm 0.1) \times 10^{9}$ & $(1.7 \pm 0.2) \times 10^{2}$ \\
\hline $4 \%$ doped & $1.1 \pm 0.05$ & $408 \pm 2$ & $(8.6 \pm 0.2) \times 10^{-11}$ & $(8.1 \pm 0.1) \times 10^{6}$ & $(3.5 \pm 0.1) \times 10^{2}$ \\
\hline
\end{tabular}

a $1 \mathrm{eV} \approx 1.6022 \times 10^{-19} \mathrm{~J}$.

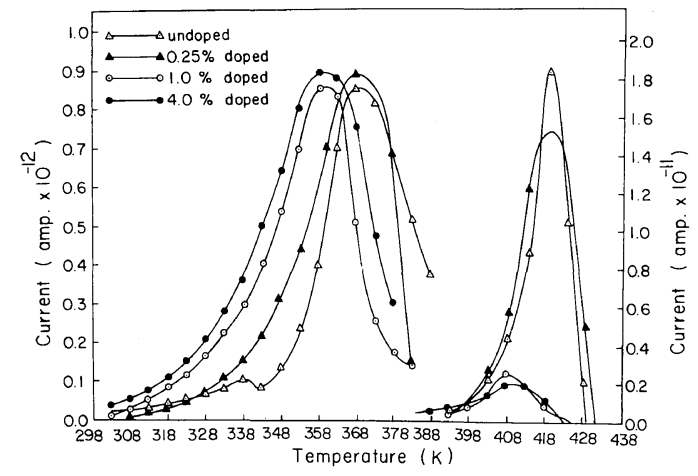

Figure 6. TSD current $v s$. temperature for undoped and chloranil-doped PS with different doping concentration under identical conditions. Vertical scale for $\beta$ and $\alpha$ peaks should be read on left handed side and for $\rho$ peak on right handed side.

\section{DISCUSSION}

\section{TSD of Pure PS}

PS is a linear polymer, whose commercial product is atactic, i.e., it is mostly amorphous. Its glass transition temperature lies in the range $363-373 \mathrm{~K}$. Besides a glass transition involving the motions of

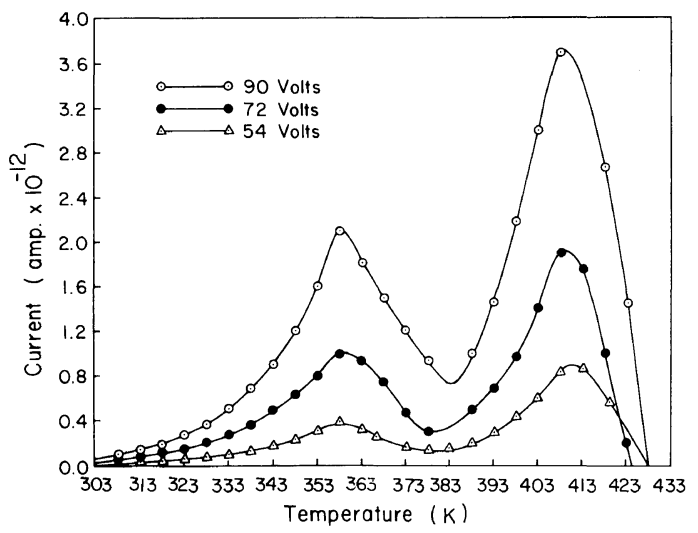

Figure 7. Current vs. temperature for $4 \%$ doped PS at different polarising fields.

chain segments of about 50 to 100 backbone atoms, two other important transitions occuring below and above the glass-transition temperature have also been reported for amorphous polymers. ${ }^{7}$ The former is due to the motion of sidegroups and the latter involves the entire chain motion. The temperature and frequency dependance of the real and imaginary parts of the dielectric constant in commercial PS for both bulk and thin film samples show the presence of 
TSD Study of Pure and Chloranil Doped Polystyrene Films

two transitions, termed $\beta$ and $\alpha$ relaxations. ${ }^{8,9}$ The $\beta$ relaxation process is observed above room temperature, for PS around $340 \mathrm{~K}$, and also has been confirmed from mechanical relaxation measurements of bulk PS, ${ }^{10}$ while the $\alpha$ transition occurs at the glass transition temperature of the polymer.

Glarum $^{11}$ has reported that trapping of charge carriers in nonpolar media can give rise to induced dipoles. Three possible trapping sites: (i) single molecule, (ii) chainfolds, and (iii) interfaces between crystalline and amorphous regions, have been reported for most polymers. ${ }^{1}$ If charge trapping takes place at these sites during a polarisation cycle of the sample, TSD spectra of such samples will have their peaks at the temperature for which thermal energies become sufficient to detrap the charges from these sites. The low temperature or $\beta$ relaxation at $338 \mathrm{~K}$ in the present measurement and at $340 \mathrm{~K}$ reported $^{12}$ for dielectric relaxation leads one to conclude that these trapping sites are contained in a region where mobility for molecular motion is great. Such regions are amorphous regions of polymers where the rotational frequency for molecular sites is large. One will, therefore, expect a low activation energy and low relaxation time for the $\beta$ TSD peak. The activation energy of $0.23 \mathrm{eV}\left(0.37 \times 10^{-19} \mathrm{~J}\right)$ and the low relaxation time, $2.2 \times 10^{3} \mathrm{~s}$, obtained in our experiment for this peak seem to be in accord with the above mechanism and agree well with the findings of other workers.

The $\alpha$ peak in the present TSD spectra occurs at about $368 \mathrm{~K}$, which lies in the region of the glasstransition temperature of PS. The $\alpha$ peak, as mentioned earlier, is due to segmental motion of the main chain and requires a higher activation energy. The activation energy of $1.13 \mathrm{eV}\left(1.81 \times 10^{-19} \mathrm{~J}\right)$ and relaxation time of $9.0 \times 10^{5} \mathrm{~s}$ observed in the present measurement for this peak are comparable to those reported for the phase transition process in PS. ${ }^{2}$ Such a phase transition will involve the entire polymer leading to a uniform polarisation. The evidence for uniform polarisation is the linear relationship between peak current and the polarising voltage (Figure 8).

The third peak, called the $\rho$ peak in the present findings and not reported in dielectric relaxation measurements, appears to be due to a release of space charges trapped around the inhomogeneities present in the sample. Such inhomogeneities are abundantly present in polymers due to their partial crystalline

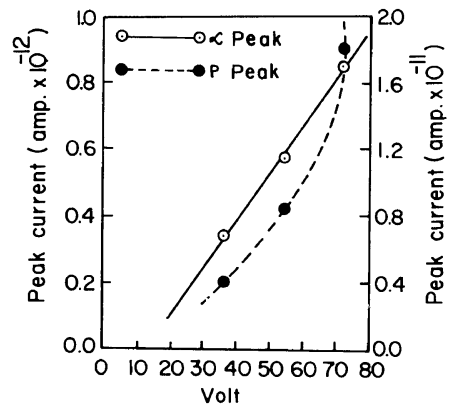

Figure 8. Peak current $v s$. voltage for $\alpha$ and $\rho$ peaks of undoped PS.

and amorphous structure. The charges are accumulated during the forming cycle of the sample at these interfaces because of the unequal ohmic conduction current arriving there. When such a sample is heated, the charges stored are neutralised by new carriers of opposite polarity that are conveyed to the interface by conduction currents which are created by the interfacial charges themselves. This neutralisation will be transient in nature, thus leading to a characteristic peak in the high temperature region. The high activation energy of $2.3 \mathrm{eV}\left(3.69 \times 10^{-19} \mathrm{~J}\right)$ and relaxation time of $3.2 \times 10^{13} \mathrm{~s}$ compare well with those reported for ohmic conduction. Excess charge polarisation gives rise to nonuniform polarisation. Our results showing a nonlinear relationship between peak current and polarising field (Figure 8) also supports the existence of nonuniform polarisation responsible for the $\rho$ peak.

\section{Effect of Doping}

Chloranil, known as tetrachloro- $p$-benzoquinone, is a weak electron acceptor organic substance with an electron affinity of $1.4 \mathrm{eV}\left(2.24 \times 10^{-19} \mathrm{~J}\right)$, whereas PS is an electron donor-type polymer. So, it is very likely that chloranil may form donor-acceptor complex with PS when mixed with it under suitable conditions. Such complexes have been reported ${ }^{13}$ to be formed by PS when a suitable electron acceptor is introduced to it. The concept of formation of donor-acceptor complexes or charge-transfer complexes (CTC's) has been supported by various workers ${ }^{14-16}$ for different polymers when iodine, which is also an electron acceptor substance, is impregnated in them. Some type of electrostatic interaction takes place between donor and acceptor atoms inside the polymer during CTC formation, the mechanism is still not very clear. However, the findings of earlier 
workers and those of the present experiment reveal that the presence of acceptor impurity atoms has a three-fold effect. Firstly, they may provide conductive pathways for the charge carriers through the noncrystalline regions, secondly they may prevent the trapping of charge carriers on the particular sites in the molecular chains, and lastly they may enhance the mobility of charge carriers. Wintle ${ }^{17}$ has also reported that the presence of electronegative groups in the polymer chain leads to progressively weaker trapping of charge carriers. In the light of the above findings we are in a position to explain the modifications observed in the TSD spectra of PS after doping it with chloranil.

The complete disappearance of the $\beta$ peak in TSD spectra of doped PS once again confirms that this peak has its origin in trapping sites present in noncrystalline regions. In doped PS these sites disappear because the dopant molecules make a link of polymer chains in the amorphous regions. The shift of both $\alpha$ and $\rho$ peaks towards lower temperature side by about $283 \mathrm{~K}$ after doping may be explained as due to the increase in mobility of charge carriers. Davies ${ }^{15}$ has also observed that introduction of iodine into polyethylene causes a mobility increase of about 4 orders of magnitude. Hence it may be thought that the presence of chloranil in the structure of PS facilitates the transitions of charge carriers and thereby less thermal energy is required to detrap them from their trapping sites. This hypothesis is further supported by the lowering of the activation energies and relaxation times, as can be seen from Table I.

The positions and magnitudes of $\alpha$ and $\rho$ peaks in $0.25 \%$-doped PS are nearly identical to their counterparts for pure PS; this suggests that a low concentration of dopant does not have any appreciable effect in modifying the characteristic properties of PS. At the same time it is obvious from the almost equal magnitudes obtained for $\alpha$ peaks in the cases of undoped and doped PS of all dopant concentrations (Figure 6) that this peak originates from the segmental motion of main chains. The effect of doping is only to make the movement of segments somewhat easier, causing the $\alpha$ peak to appear at a lower temperature. The progressively lower magnitudes observed for the $\rho$ peak on increasing the dopant concentration (Figure 6) can again be ascribed to successive loss in accumulation of space charges at the interfaces as the chloranil occupies them.
However, it can't be said conclusively whether chloranil is present there in molecular form or ionic form. Rather extensive studies are needed to draw definite conclusions in this regard.

\section{CONCLUSIONS}

(1) $\beta$ peak in TSD spectra of pure PS originates from trapping sites present at particular atoms or molecules contained in noncrystalline regions.

(2) The $\alpha$ peak occurs because of segmental motions of main chains.

(3) The third peak in present investigation is the space charge peak and appears due to the accumulation of charge carriers at the crystalline amorphous interfaces.

(4) Chloranil forms CTC's with PS and has a three-fold effect: (a) to provide conductive pathways for the charge carriers through noncrystalline regions; (b) to prevent the trapping of charge carriers; (c) to enhance their mobility.

Acknowledgement. One of the authors (Kulshrestha) expresses his thanks to University Grants Commission of India for the award of a Teacher fellowship under its faculty improvement program.

\section{REFERENCES}

1. J. van. Turnhout, Ed., "Thermally Stimulated Discharge of Polymers Electrets," Elsevier, Amsterdam, Oxford, and New York, N.Y., 1975.

2. M. M. Perlman, J. Electrochem. Soc., 119, 892 (1972).

3. S. N. Prasad and R. S. Prasad, Ind. J. Phys., 49, 596 (1975).

4. M. M. Perlman and R. A. Creswell, J. Appl. Phys., 41, 62365 (1970).

5. G. F. J. Garlick and A. F. Gibson, Proc. Phys. Soc., 60, 574 (1948).

6. J. van. Turnhout, Polym. J., 2, 173 (1971).

7. A. D. Jenkins, Ed., "Polymer Science," Vol. II, N. Holland Publishing Co., Amsterdam, 1972.

8. T. Hara, M. Nozaki, and S. Okamoto, Jpn. J. Appl. Phys., 6, 1138 (1967).

9. B. Bhargava, Ph.D. Thesis, University of Saugar, India, 1977.

10. I. Jenckel, J. Polym. Sci., A-2, 41, 528 (1965).

11. S. H. Glarum, J. Phys. Chem. Solids, 24, 1577 (1963).

12. N. Saito, K. Okano, S. Iwayanagi, and T. Hideshima, "Solid State Physics," Vol. 14, F. Sertz and D. Turnbull, Ed, Academic Press, New York, N.Y., 1963, p 387.

13. W. Slough, Trans. Faraday Soc., 58, 2360 (1962). 
TSD Study of Pure and Chloranil Doped Polystyrene Films

14. K. Jain, A. C. Rastogi, and K. L. Chopra, Phys. Stat. $\quad$ 16. P. C. Mehendru, K. Jain, and P. Mehendru, J. Phys. Solidi (A), 20, 167 (1973).

15. D. K. Davies, J. Phys. D., Appl. Phys., 5, 162 (1972). 17. H. J. Wintle, J. Acoust. Soc. Am., 53, 1578 (1973). 